CERN LIBRARIES, GENEVA

CERN-EP/84-162

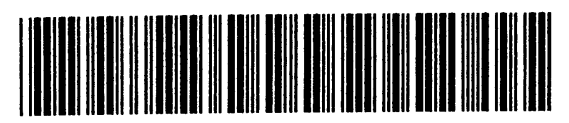

29 November 1984

CM-P00071039

\title{
PROTOTYPE TESTS OF TWO CONTROLLED GEOMETRY DRIFT CHAMBERS
}

D. Bettoni ${ }^{+}$, B. Dolgoshein*, C.W. Fabjan, H. Hofmann, J. Perez, F. Piuz,

P. Quéru, V. Radeka**, E Rosso, A. Rudge, D. Soria-Buil, J.P. Vanuxem and W.J. Willis.

CERN, Geneva, Switzerland

\begin{abstract}
$\underline{\text { ABSTRACT }}$
This report presents results on the performance of two new drift chamber geometries, which were optimized for space resolution and two-track separation. The best performance requires isochronous drift collection, low electron diffusion, and careful signal shaping. The role of the mobility of electrons and positive ions is emphasized.

We tested two geometries, and at a gas pressure of 1 atm we found $\sigma \simeq 60-70 \mu \mathrm{m}$ for the single-track resolution and $\Delta \simeq 600 \mu \mathrm{m}$ for the two-track separation.
\end{abstract}

Submitted to Nuclear Instruments and Methods

+ Visitor for Syracuse University, Syracuse, N.Y., USA

* Visitor from Moscow Institute of Engineering Physics, Moscow, USSR

** Visitor from Brookhaven National Laboratory, Upton, USA. 


\section{INTRODUCTION}

The continuous efforts that are being made to improve and to miniaturize detectors of high-energy particle collisions, and the increasing importance of being able to recognize reliably short-lived particles through their decay vertices, has stimulated the development of track detectors, which should have the following properties:

- excellent single-track resolution, $\sigma<50 \mu \mathrm{m}$;

- very good two-track separation, $\Delta \leq 500 \mu \mathrm{m}$;

- operation in a high multiplicity environment and frequently at high event rates.

We are developing imaging drift chambers which will have such properties, and are using construction techniques which should allow the realization of spectrometer or storage ring chambers of considerable size [1]

A number of parameters have to be carefully optimized [2] to achieve such a level of performance; we single out the most critical ones:

- Diffusion of the ionization electrons along the drift path affects both the single-track precision and two-track separation. It can be reduced by increasing the operating pressure, $p: \sigma_{\text {diff }}^{2} \sim(1 / p) \cdot f(E / p)$; and by using 'cool' gases, such as $\mathrm{CO}_{2}, \mathrm{NH}_{3}$, or $\mathrm{C}_{2} \mathrm{H}_{6} \mathrm{O}$, where the characteristic energy $\varepsilon$ of the electrons is close to the thermal limit $\varepsilon$ $\sim \mathrm{kT}$. In our measurements $\mathrm{CO}_{2}$ was used.

- Time differences between different ionization clusters belonging to the same track and arriving on the same wire have to be minimized for best two-track separation. We achieved approximately such an 'isochronous' operation by reducing the track segment accepted for the position measurement and therefore the corresponding drift-time differences.

- For better two-track separation, the signal has to be carefully shaped to cancel the positive ion tail [3]. 


\section{DESIGN CONSIDERATIONS}

The mechanical and the electrical layout of the two chamber geometries were first computer-modelled [4]. The electric field was calculated using a Green's function technique; knowledge of the field distributions allowed calculation of the electron trajectories, evaluation of the drift-time differences of various track segments arriving at the same wire, and estimates of the gas gain based on the measured properties of the chamber gas $\mathrm{CO}_{2}$ and for a variety of potential distributions.

The 'restricted drift' geometry was implemented in two mechanically very different ways.

Figure 1 is a scale drawing showing the layout of the 'wire chamber' geometry. In this geometry the length of the accepted track segments is predominantly determined by the potential $V_{c}$ on the 'channelling' wires C. The wire $P$ serves to decouple adjacent sense wires; 'guard' wires $G$ aid in the gain control on the sense wires, and each guards its nearest sense wire from ionization electrons of tracks on the side of the guard wire. Consequently, this geometry is characterized by the absence of the 'right-left' ambiguity and hence the need for 'right-left' pattern recognition. The track acceptance is defined electrically by the channelling wires C (Fig. 1). An example of the electrostatic field calculation is shown in Fig. 2, giving the drift paths (dotted lines) together with the equal arrival-time curves (solid lines); for the case shown, the spacing between the sense wires is $12 \mathrm{~mm}$, and each sense-wire collects ionization from a $3.5 \mathrm{~mm}$ long track segment, corresponding to a maximum spread in drift times from this segment of $80 \mathrm{~ns}$ for the case of drift in $1.5 \mathrm{kV} / \mathrm{cm}$ in $\mathrm{CO}_{2}$ and for tracks parallel to the sense wire plane. This programme was also used to minimize the field non-linearities along the chamber boundaries and to estimate the gas gain.

The 'wire' chamber was constructed using the well-tested techniques employed, for example, for the central detector of the Axial Field Spectrometer (AFS) [5]. The wires are held and positioned by being crimped in a metal tube fixed in a plastic insert. The inserts fit into holes drilled in the chamber end-plates. High-accuracy wire positioning is made 
possible by drilling the end-plate holes with $5 \mu \mathrm{m}$ precision relative to each other. The $20 \mu \mathrm{m}$ diameter sense wires were positioned to an accuracy of $\sigma<10 \mu \mathrm{m}$, using a cross-crimping method which has been developed to centralize the wires [1].

The second, restricted geometry, the 'accordeon' chamber was constructed by folding a $25 \mu \mathrm{m}$ thick aluminium foil around $300 \mu \mathrm{m}$ wires, positioned as above to form the desired electrode geometry (Fig. 3 ). In this geometry the track acceptance is defined mechanically and only two operating voltages are required. Quartz windows were fitted to both chambers to enable laser illumination of the entire active area.

\section{ELECTRONICS}

The electronics chain uses a grounded base preamplifier [6], driving a main amplifier/discriminator with a bandwidth of $60 \mathrm{MHz}$ [7]. Crucial for optimum two-track separation is the pulse shaping of the signal current

$$
\left.i(t)=\frac{Q_{a} \cdot v^{+}}{r_{a} K} \cdot \frac{1}{1+(t / t g}\right) ; \quad t_{0}=\frac{2 r_{a}}{3 v^{+}}, k=2\left(\frac{\pi L}{s}-\log \frac{2 \pi r_{a}}{s}\right)
$$

where $Q_{a}$ is the avalanche charge, $\mathrm{r}_{a}$ the radius of the anode wire, $\mathrm{E}_{\mathrm{a}}$ the electric field at the anode, and $\mathrm{v}^{+}$the positive ion velocity at the field $E_{a}$. The parameter $2 L$ specifies the chamber gap and $s$ the separation between wires. Also critical is the value of the positive ion velocity $\mathrm{v}^{+}$, which sets the scale for the long tail in the signal current. We employed a differentiating network with a characteristic time constant of $\tau \simeq 20$ ns to cancel this positive ion tail. Proper cancellation of both the preamplifier time constant and the positive ion tail requires two pole/ zero networks [3,8]. Our simplified shaping was optimized for the detection of close-by tracks, although the second pulse was registered with reduced efficiency. A fast shift-register routes each timing signal into four TDCs (LeCroy 2228A) giving a capability of four hits per wire. Six wires were instrumented in this way. A single $100 \mathrm{MHz}$ sampling-rate transient recorder (LeCroy TR8818 and MM8103/8) is used to investigate the pulse shape; it can be connected to any wire, before or after the single pole filter. 
The effect of the electron drift velocity (which determines the arrival of clusters from the same track and from neighbouring tracks), the positive ion drift velocity, and the signal-shaping wire were studied in detail. Cluster arrival times were calculated with the field program described in Section 2, and the convolution of the chamber signal with the electronics response was simulated with the circuit analysis program SCEPTRE [9]. This simulation indicated that e.g. two tracks, separated by $500 \mu \mathrm{m}$ would not be separated, if the accepted track length were $7 \mathrm{~mm}$, but $2 \mathrm{~mm}$ long track segments, spaced by $500 \mu \mathrm{m}$, should be resolved.

\section{RESULTS USING A LASER}

Extensive investigations were carried out on the 'wire' chamber using a nitrogen laser $(\lambda=337 \mathrm{~nm})$ to determine the following quantities:

a) drift velocity versus electric field;

b) position resolution versus drift field and drift distance;

c) electric-field uniformity as monitored by the drift time as a function of drift distance;

d) information on the double-track resolution.

For these measurements the chamber was operated with $100 \% \mathrm{CO}_{2}$ at a gas gain of $\sim 4 \times 10^{4}$ (measured with a $300 \mathrm{~ns}$ integration time using a ${ }^{55} \mathrm{Fe}$ source). The laser beam had a divergence of $1 \mathrm{mrad}$ and was collimated with a $1 \mathrm{~mm}$ diameter pinhole. The primary signal collected from a $3 \mathrm{~mm}$ laser track segment was measured to correspond to approximately 100 electrons, i.e. the laser was producing approximately 10 times the ionization density compared with that from a minimum ionizing particle [3].

The results for spatial resolution are given in Fig. 4, showing the r.m.s. deviation of the position obtained with one wire (not used in the fit) relative to a track, defined by a least squares fit to the position measurement of four wires. A resolution of $\sigma=50 \mu \mathrm{m}$ was obtained for tracks at a distance of $8 \mathrm{~mm}$ from the sense wires. The small additional uncertainty on this four-wire track definition was not unfolded in the single-wire resolution shown in Fig. 4. In Fig. 5 we show the dependence of drift velocity and resolution as a function of the electric drift field. The value of $\mathrm{E}_{\mathrm{dr}} \simeq 1.5 \mathrm{kV} / \mathrm{cm}$ corresponds to optimum resolution, consistent with the minimum in diffusion for $\mathrm{CO}_{2}$ at this value of the electric field. 
For the tests on two-track separation, a mirror with $80 \%$ reflectivity was placed behind the chamber to reflect the laser beam back through the chamber. The observed amplitude of the reflected track signal was $\sim 50 \%$ of that of the incident track.

Reconstruction efficiency (determined as four out of five points on each track) is plotted against track separation in Fig. 6. At $500 \mu \mathrm{m}$ separation, $50 \%$ of the double tracks are resolved. The transient recorder registration of the signals before and after the pulse shaping network for two tracks with $500 \mu \mathrm{m}$ separation are shown in Fig. 7.

\section{RESULTS WITH MINIMUM IONIZING PARTICLES}

Both chambers were tested in the high rate environment of the CERN Intersecting Storage Rings (ISR). For the wire chamber, silicon-strip detectors were installed in front of and behind the chamber in order to define multitrack events accurately (Fig. 8).

The 'accordeon' chamber was operated with a gas gain of $\sim 5 \times 10^{4}$ (measured with a 300 ns integration time using a ${ }^{55} \mathrm{Fe}$ source) and an accepted track length of $\sim 3 \mathrm{~mm}$, the wire chamber with a gas gain of $\sim 1.3 \times 10^{4}$ and an accepted track length of $\sim 3.5 \mathrm{~mm}$. Both chambers were operated with drift fields of $\sim 1.5 \mathrm{kV} / \mathrm{cm}$ and with a $\mathrm{CO}_{2} / \mathrm{C}_{2} \mathrm{H}_{6}$ (90/10) mixture. The $10 \%$ ethane mixture was required to reduce the afterpulsing in the 'accordeon' chamber, probably caused by secondary emission from the aluminium cathode of that chamber.

First we present and discuss results on single-track accuracy. The performance of the accordeon chamber for different drift distances and for three different levels of the drift field is summarized in Fig. 9. For these measurements, straight tracks were fitted through four points, and the deviation of the hits on a fifth central wire $\sigma_{c}$ was used to evaluate the single-track accuracy. This measured resolution can be understood as the superposition of the following principal contributions:

- diffusion as a function of drift field and drift distance;

- statistics of the effective number of electrons $n_{\text {eff. }}$ influencing the drift-time measurements;

- fitting and electronic measurement errors. 
From published measurements on diffusion in $\mathrm{CO}_{2}$ [2], the diffusion for $1 \mathrm{~cm}$ drift is estimated to be 80,70 , and $68 \mu \mathrm{m}$ for $1.2,1.5$, and 1.9 $\mathrm{kV} / \mathrm{cm}$ fields, respectively.

The number $n_{\text {eff }}$ of electrons effectively contributing to the drifttime measurement is smaller than the number of electrons produced along the track within the drift field acceptance of the sense wire. This is due to the fact that the drift collection in our geometry is not perfectly isochronous and results in a spread of arrival times at the anode wire ('geometrical dispersion'). The number $\mathrm{n}_{\text {eff }}$ may be estimated by assuming contributions from an effective track segment for which the geometrical drift-time dispersion due to non-isochronism is comparable to the dispersion $\sigma_{\text {diff }} \simeq 70 \mu \mathrm{m}$ due to diffusion. Inspection of the electrostatic field map (Fig. 2) indicates that the equivalent geometrical dispersion $\sigma_{\text {geom }} \simeq 6$ ns corresponds to an effective track length of ح $2 \mathrm{~mm}$. With an average of 30 primary ionization electrons produced per centimetre, about 5-8 electrons contribute effectively to the measured resolution (except for a few per cent where long-range $\delta$ electrons are produced) [3]. It is estimated that:

$$
\sigma_{1}(\text { trigger on first electron arrival })=\left(\pi \sqrt{2 \sqrt{3 \ln n_{e f f}}}\right) \sigma \text {, }
$$

where $\sigma=\left(\sqrt{ } \sigma_{\text {geo }}^{2}+\sigma_{\text {diff }}^{2}\right)=\sigma \operatorname{diff} / 2$;

hence $\sigma_{1}=\left(\pi \cdot \sigma_{\text {diff }}\right) /\left(\sqrt{6 \ln n_{\text {eff }}}\right) \simeq 67 \mu \mathrm{m}$.

The contribution of the electronics ( $1 \mathrm{~ns}$ digitization) is negligible and the error on the track reconstruction contributes $\sigma_{\text {total }}^{\text {estimated }}=\sqrt{5 / 4} \sigma_{1}$ $=75 \mu \mathrm{m}$, which is to be compared with the measurements. We conclude that the measured single-track accuracy can be explained by the diffusion properties of the drift gas and plausible assumptions regarding the statistics of the collected electrons. In Fig. 10 we show the experimental results obtained with the drift chamber using the multiwire focusing geometry (Fig. 1); these are comparable to the accordeon results, to which the above breakdown of the various contributions is also applicable. It should be noted that the simple estimate of the contribution of diffusion does not include the perhaps substantial effects on $\sigma_{\text {diff }}$ and the electron and ion drift velocity due to the very large ratio $\mathrm{E} / \mathrm{p}$ near the anode wires, for which no explicit measurements exist. 
In the measurements on the two-track resolution, we used two planes of silicon-strip detectors ( $70 \mu \mathrm{m}$ strip separation) and required matching hits in these detectors. Again, for each reconstructed track, a minimum of four hit wires out of six were required. The results are histogrammed in Fig. 11 and show the minimum separation of two hits on a wire belonging to a pair of reconstructed tracks. An estimate of the reconstruction efficiency in the drift chamber would have required independent track reconstruction in the Si-strip detectors, which unfortunately was not possible with the instrumentation available to us. Whilst we cannot quote an absolute efficiency for two-track reconstruction, we believe that the distribution in two-track separation (Fig. 11) is consistent with a two-track reconstruction efficiency constant for track separations $\Delta \geq$ $700 \mu \mathrm{m}$. This is based on the fact that such a distribution would be expected, as shown by the solid line in Fig 11. Its computation is based on the measured track distribution across the $12 \mathrm{~mm}$ wide area of the drift gap and assuming the same efficiency distribution for two-track reconstruction as was observed in the laser data (Fig. 6). Measurements done under identical conditions but with a standard gas mixture, $\mathrm{Ar} / \mathrm{C}_{2} \mathrm{H}_{6}(50 / 50)$, indicated that full efficiency was only obtained for track pairs with $\Delta \geq 2 \mathrm{~mm}$.

Several parameters related to the drift-time difference between neighbouring tracks (i.e. drift velocity) and the pulse shape (i.e. positive ion velocity) need to be controlled to approach the theoretical performance:

i) The choice of gas. Electrons drift in $\mathrm{CO}_{2}$ in fields of $1-2 \mathrm{kV} / \mathrm{cm}$ at a velocity of approximately $20 \%$ of that in a standard saturated argon/ ethane mixture ( $\sim 12 \mu \mathrm{m} / \mathrm{ns}$ compared to $\sim 50 \mu \mathrm{m} / \mathrm{ns})$. It is the electron drift velocity which determines the time difference of avalanches from close-by tracks. The positive ion velocity, which governs the time dependence of current pulse, is however only $30 \%$ slower in $\mathrm{CO}_{2}$ compared with an argon/ethane mixture. This favourable ratio $v_{\mathrm{dr}}^{\mathrm{el}} / \mathrm{v}_{\mathrm{dr}}^{\text {ion }}\left(\mathrm{CO}_{2}\right) \simeq 0.3 \mathrm{v}_{\mathrm{dr}}^{\mathrm{el}} / \mathrm{v}_{\mathrm{dr}}^{\text {ion }}$ (argon/ethane) is a prerequisite for the improved double-track resolution. 
ii) Pulse shaping ('pole/zero' cancellation) and near-isochronous operation. The pulse shaping of the positive ion signal needs to be optimized as a function of the ion velocity. This is relatively simply accomplished after the preamplifier, using two differentiations with pole/zero cancellations. One of these cancels the tail arising in the preamplifier, and the other cancels the tail due to positive ion motion. A very narrow overall response can be achieved to signals arising from localized ionization (single clusters), with a nearly Gaussian response of about 10-15 ns wide at the base [8]. This width should be chosen according to electron diffusion and the spread in the electron drift time.

Note, however, that besides optimum pulse shaping, near-isochronous drift collection on a time scale commensurate with the intrinsic double-track resolution is most important. Should electrons arrive over time intervals longer than the characteristic shaping time, a signal of continuous 'after-pulsing' would not allow for any improved two-track resolution.

The geometrical arguments presented here indicate that our approach is limited to tracks with nearly normal incidence with respect to the drift field lines. Specifically, the deviation $\varepsilon$ from normal incidence is limited to tg $\varepsilon \leq$ ( $\sigma_{\text {single track }}$ /effective track length), so as not to limit the single-track resolution. A similar restriction applies to double-track separation $\Delta$, where particle incidence has to be restricted within $\operatorname{tg} \varepsilon^{\prime} \leq(\Delta / 2) /($ effective track length). 
7. CONCLUSIONS

The use of a 'cool-slow' gas together with collimating geometry and pulse shaping gives a factor of 3 or more accuracy in single-track resolution and double-track separation compared with conventional designs.

In the two test chambers implementing these principles, we achieved a positional accuracy of $\leq 70 \mu \mathrm{m}$, and $\sim 600 \mu \mathrm{m}$ double- track resolution.

Second coordinate readout may be possible by printing either a delay line or 'pad' readout pattern onto the accordeon, or by substituting a thin ( $2 \mathrm{~mm}$ ) delay line in place of the guard wire electrode in Fig. 1. 


\section{REFERENCES}

[1] W.M. Evans, Proc. Int. Europhysics Conf. on High-Energy Physics, Brighton, 1983 (RAL, Didcot, 1983), p.424;

D. Bettoni et al., Prototype tests of a controlled geometry drift chamber, Contributed paper No. 295 to same Conference.

[2] S. Bobkov et al., CERN-EP/83-81 (1983) and Nuc1. Instrum. Methods (to be published).

[3] F. Sauli, CERN 77-09 (1977);

R.A. Boie et al., Proc. IEEE Science Symposium, Orlando 1980;

IEEE Trans . NS-28 (1981) 603.

F. Lapique and F. Piuz, Nuc1. Instrum. Methods 175 (1980) 297.

[4] T.J. Killian, Nuc1. Instrum. Methods 176 (1980).

[5] D. Cockerill et al., Phys. Scr. 23 (1981) 649.

[6] V. Radeka and P. Rehak, Brookhaven National Laboratory report 23345 (1977).

[7] J.C. Berset et a1., Nucl. Instrum. Methods 176 (1981).

[8] V. Radeka et al., to be published.

[9] J.C. Bowers and S.R. Sedone: Sceptre, a Computer Program for Circuit and Systems Analysis, Prentice-Hall, Inc., Englewood Cliffs, N.J. 


\section{FIGURE CAPTIONS}

Fig. 1: Mechanical layout of the "wire chamber" geometry. Channelling wires C define the track segment accepted by the anode wires, whereas the guard wires ( $x$ ) limit the track acceptance to one side of the cell.

Fig. 2: Example of an electrostatic field calculation for the 'wire' geometry. The pointed lines are electric field lines, along which electrons drift to the anode. The solid lines are the loci of isochronism, calculated for the case of $\mathrm{CO}_{2}$ at a drift velocity $\mathrm{v}_{\mathrm{dr}}=12 \mu \mathrm{m} / \mathrm{ns}$ The potentials were chosen to give a drift field $E_{\mathrm{dr}}=1.5 \mathrm{kV} / \mathrm{cm}$; the focusing wires are at $1.8 \mathrm{kV}$ to accept a track length of $\sim 3.5 \mathrm{~mm}$.

Fig. 3: Scale drawing of the 'accordeon' chamber. The 'accordeon' cathode is constructed from $25 \mu \mathrm{m}$ thick aluminium foil, held in position by $300 \mu \mathrm{m}$ diameter nylon strings.

Fig. 4: Laser measurement of spatial resolution as a function of drift distance.

Fig. 5: Laser measurements of single-track resolution (left ordinate) and drift velocity (right ordinate) as a function of drift field.

Fig. 6: Laser tests showing the double-track reconstruction efficiency as a function of track separation.

Fig. 7: Transient recorder output showing the typical current pulse (a) before and (b) after differentiation. One bin corresponds to $10 \mathrm{~ns}$.

Fig. 8: Layout of apparatus during tests in the ISR. SS1, SS2 and SS3 are silicon-strip detectors.

Fig. 9: Resolution as a function of drift distance for the 'accordeon' chamber for three drift fields (ISR test results). Errors are statistical only. 
Fig. 10 ISR test results of resolution as a function of drift distance for the 'wire chamber'. Errors are statistical only.

Fig. 11 'Wire' chamber multiple track resolution in microns using the siliconstrip detectors as beam-defining elements (ISR test results). The solid line represents our Monte Carlo estimate for this distribution, assuming constant reconstruction efficiency for $\Delta \geq$ $700 \mu \mathrm{m}$. 


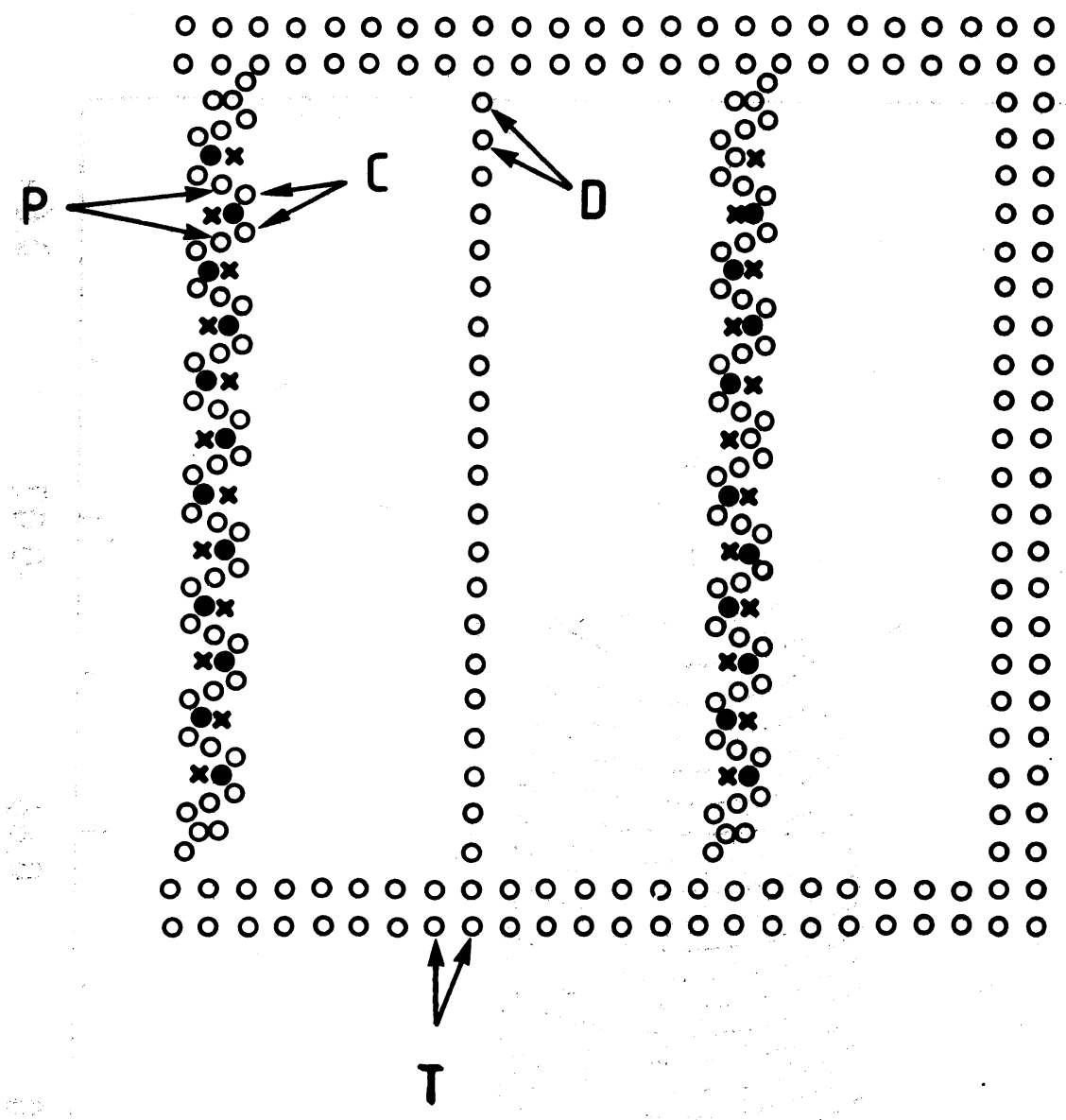

× Guard wire

(G)

- Anode wire

- Channelling wire (C)

- Potential wire

- Electric field

- Termination wire (T)

- Drift field wire (D)

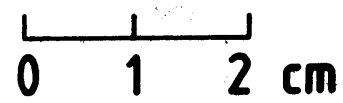

Fig. 1 


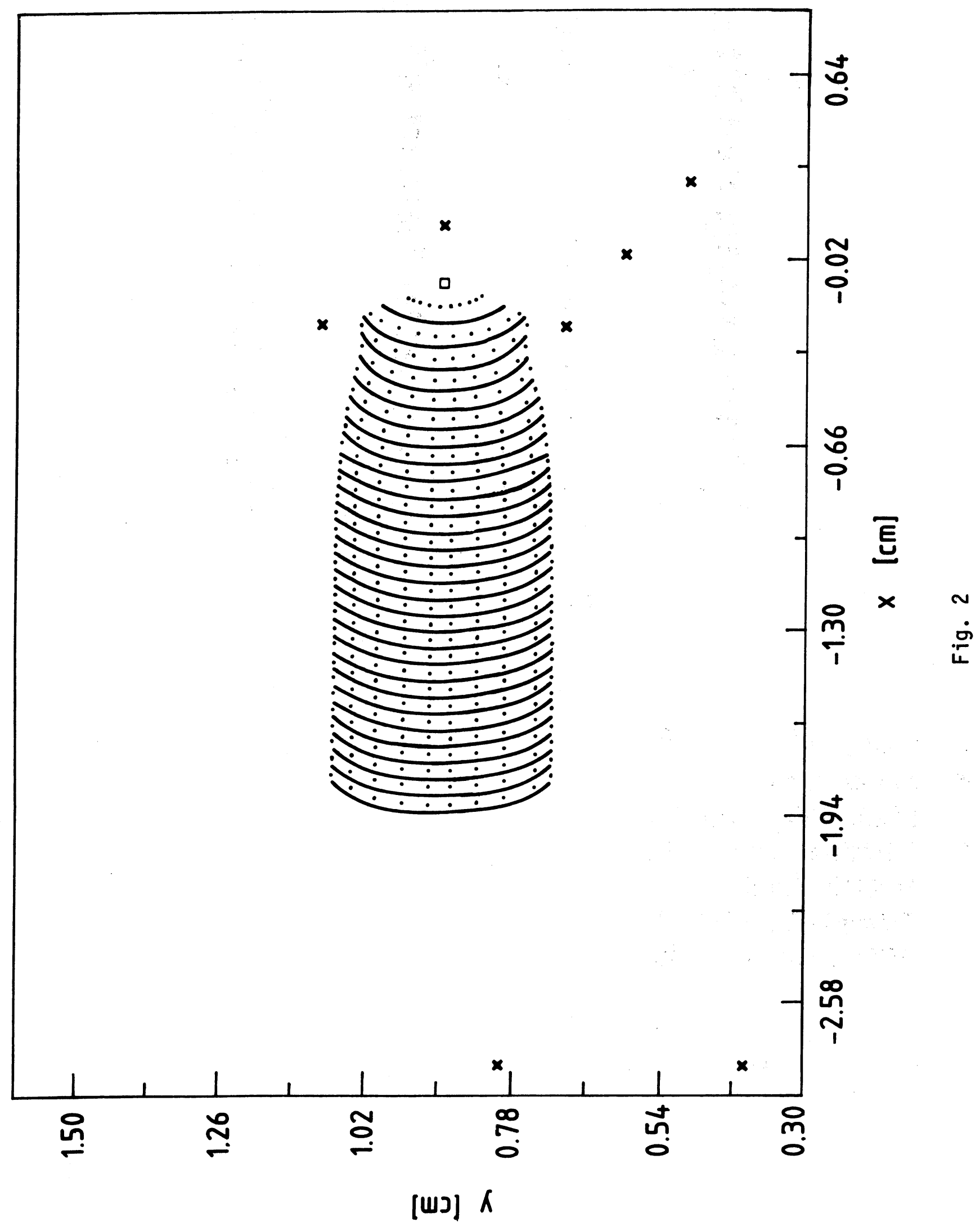


- Sense wire

- Support wire

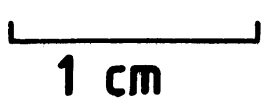

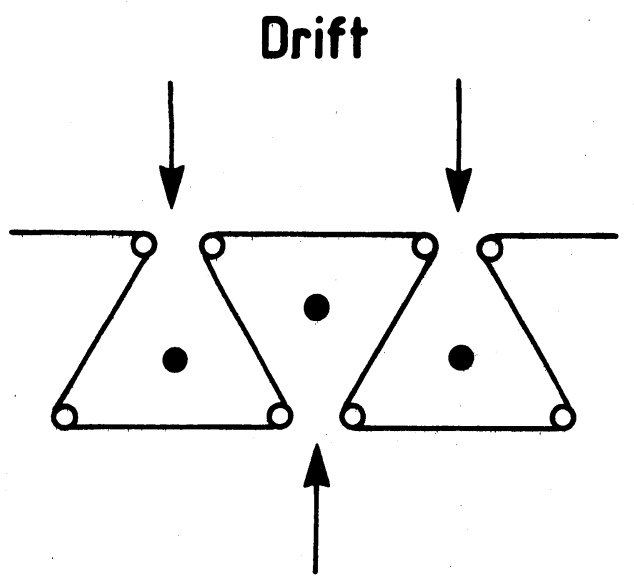

Drift

Fig. 3

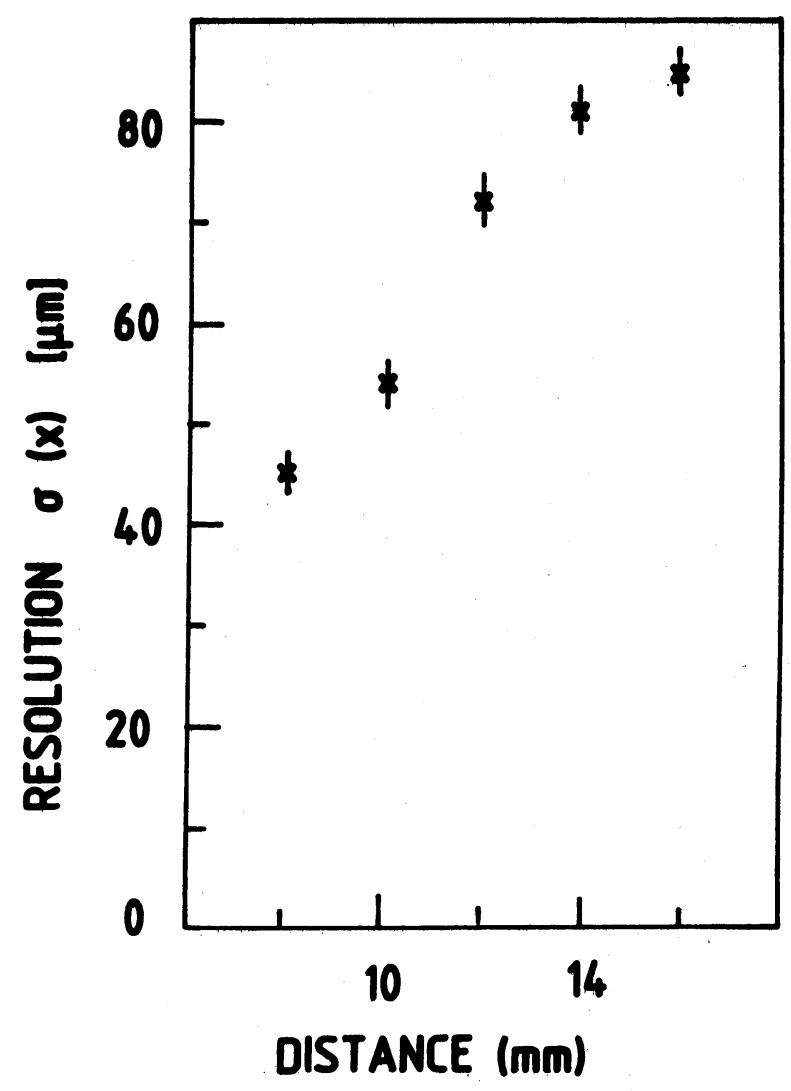

Fig. 4 


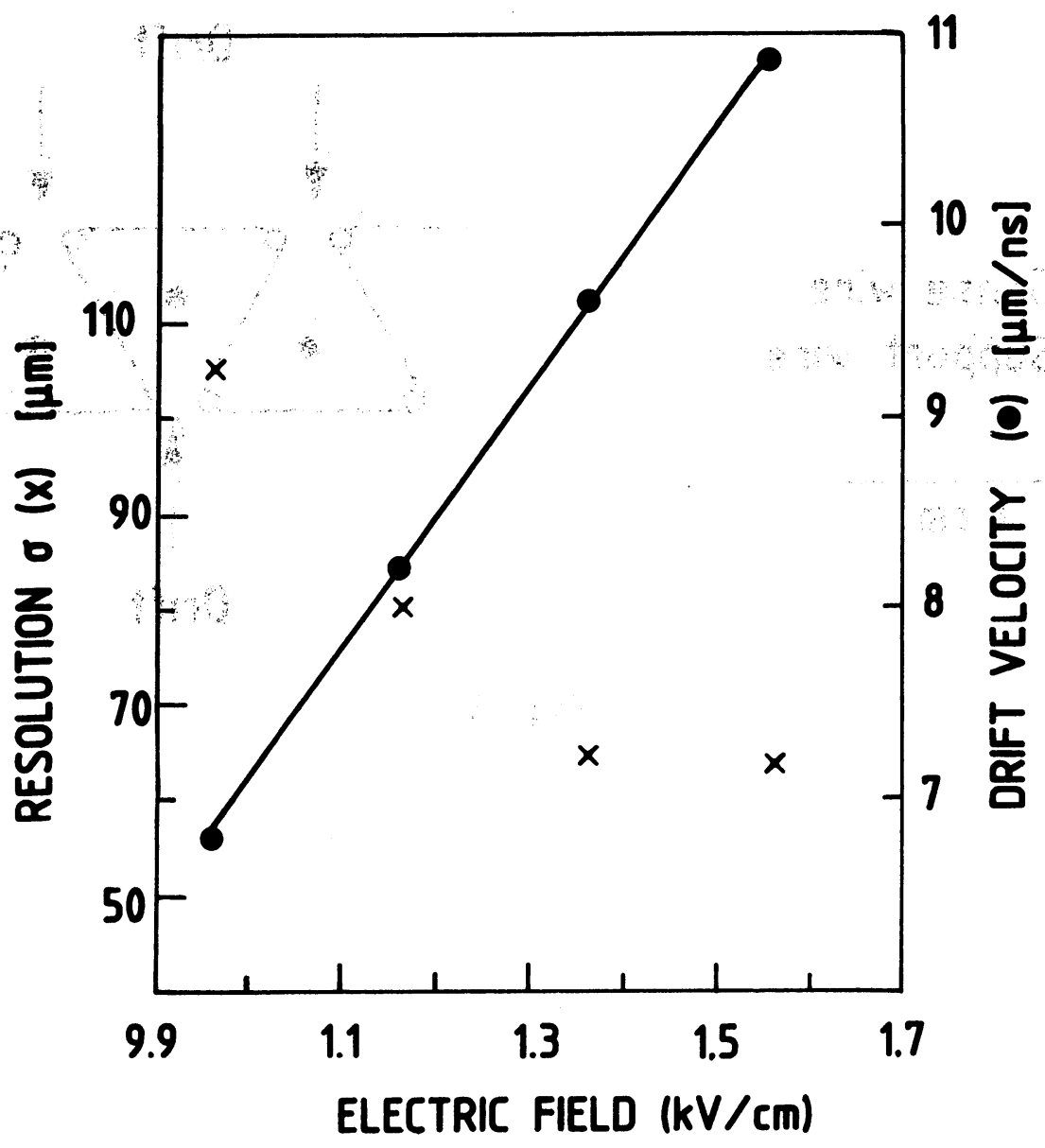

Fig. 5

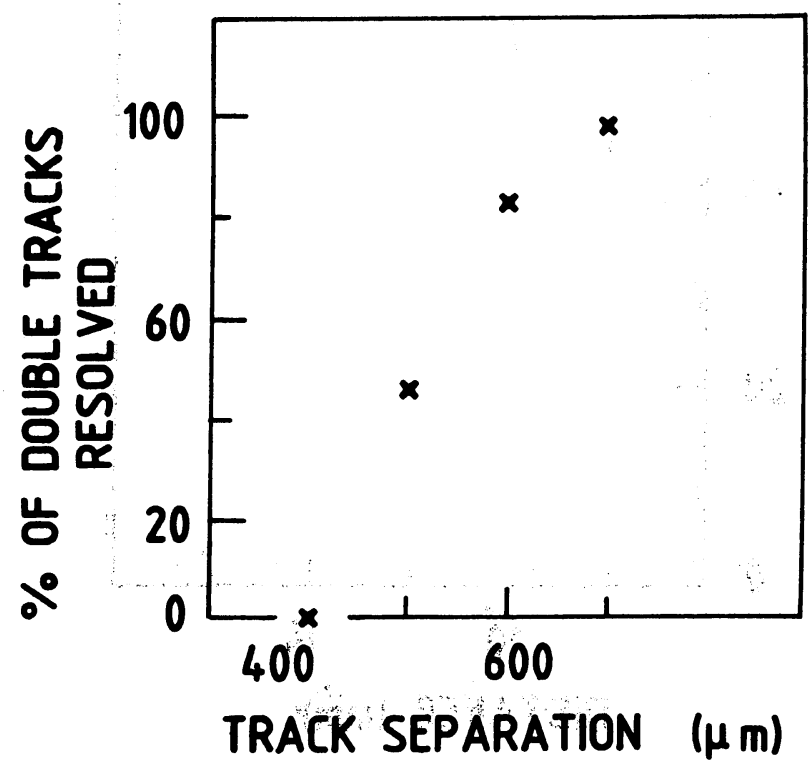

Fig. 6 


$$
E
$$




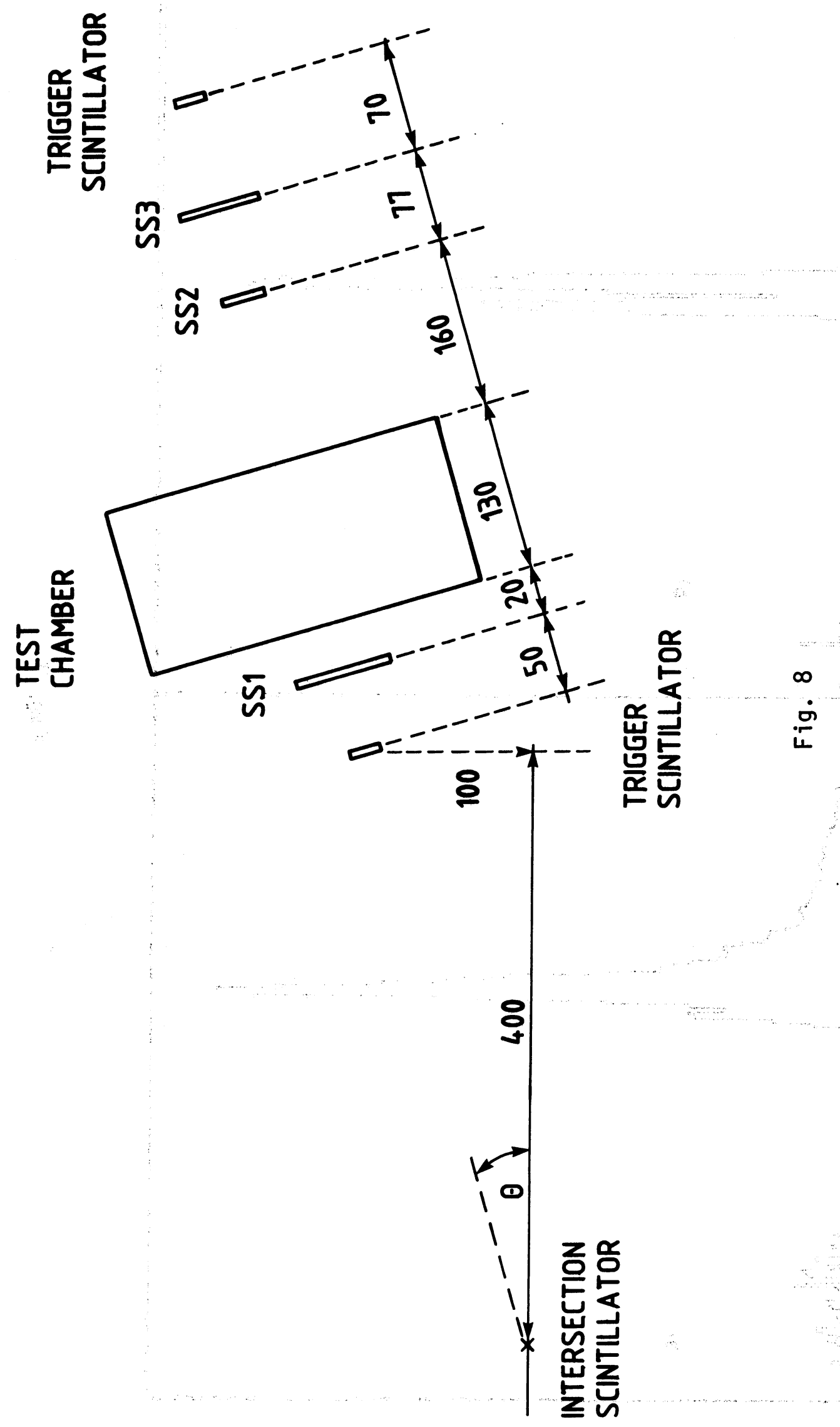



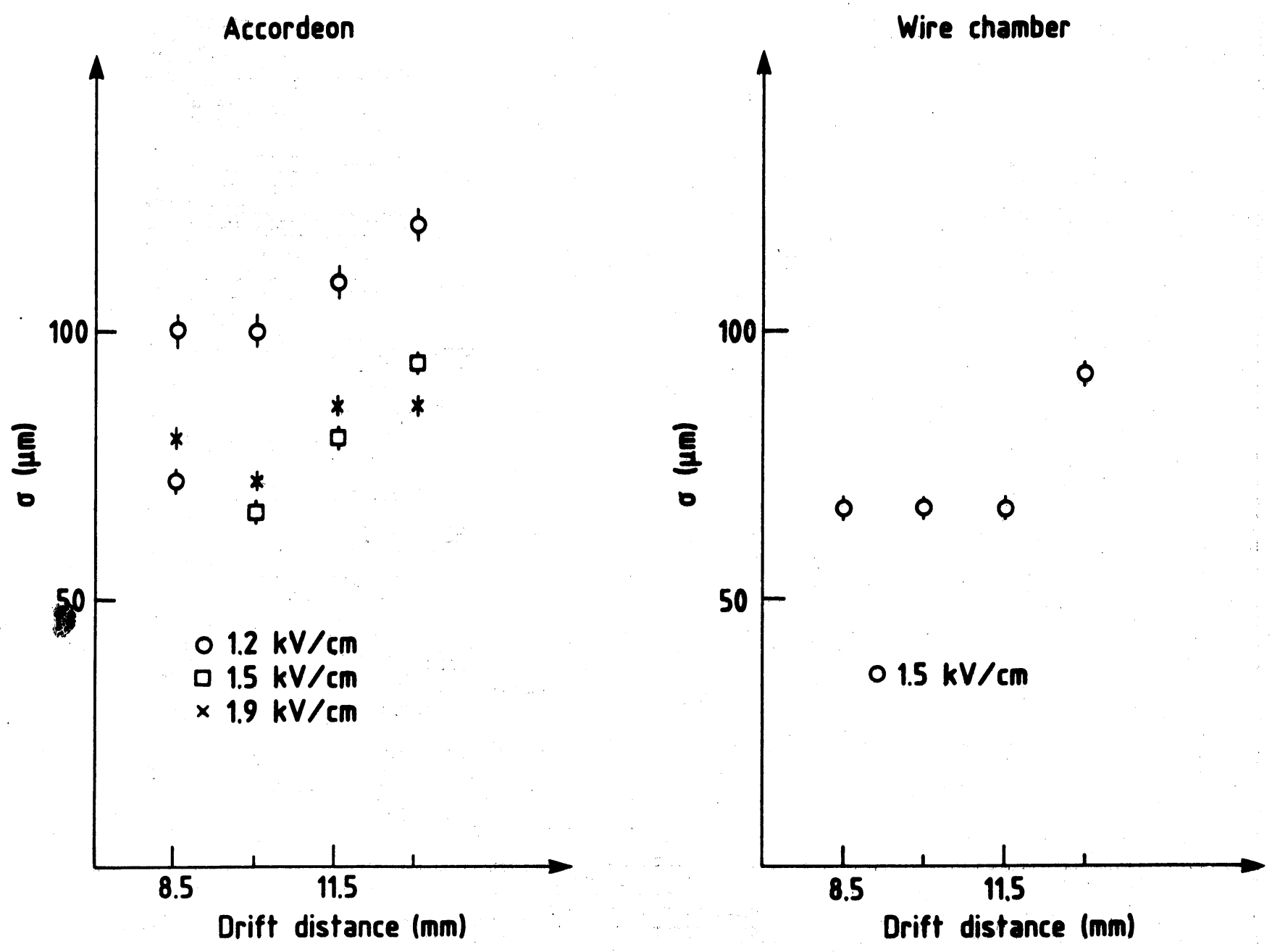

Fig. 9

Fig. 10 


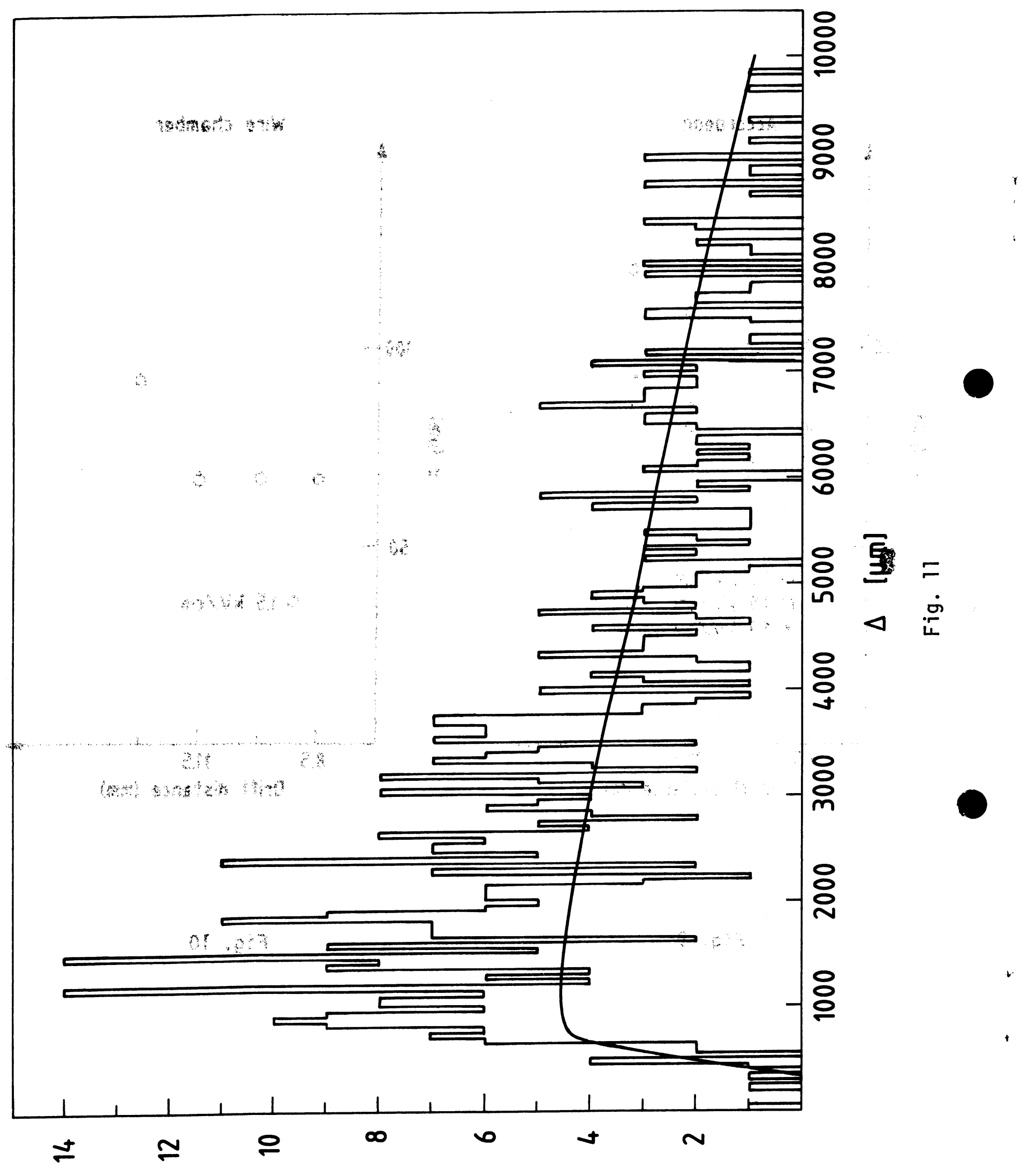

